\title{
Scandinavian Actuarial Journal
}

\section{Further developments in the Erlang(n) risk process}

\section{Agnieszka I. Bergel \& Alfredo D. Egídio Dos Reis}

To cite this article: Agnieszka I. Bergel \& Alfredo D. Egídio Dos Reis (2015) Further developments in the Erlang(n) risk process, Scandinavian Actuarial Journal, 2015:1, 32-48, DOI: 10.1080/03461238.2013.774112

To link to this article: http://dx.doi.org/10.1080/03461238.2013.774112

\section{曲 Published online: 31 May 2013.}

Submit your article to this journal 준

LIII Article views: 139

Q View related articles $\llbracket$

View Crossmark data 


\title{
Further developments in the $\operatorname{Erlang}(n)$ risk process
}

\author{
AGNIESZKA I. BERGEL and ALFREDO D. EGÍDIO DOS REIS* \\ Department of Mathematics, ISEG and CEMAPRE, Technical University of Lisbon, Lisbon, Portugal
}

(Accepted 3 February 2013)

\begin{abstract}
For actuarial aplications, we consider the Sparre-Andersen risk model when the interclaim times are Erlang $(n)$ distributed. We first address the problem of solving an integro-differential equation that is satisfied by the survival probability and other probabilities, and show an alternative and improved method to solve such equations to that presented by $\mathrm{Li}(2008)$.

This is done by considering the roots with positive real parts of the generalized Lundberg's equation, and establishing a one-one relation between them and the solutions of the integro-differential equation mentioned before.

Afterwards, we apply our findings above in the computation of the distribution of the maximum severity of ruin. This computation depends on the non-ruin probability and on the roots of the fundamental Lundberg's equation.

We illustrate and give explicit formulae for Erlang(3) interclaim arrivals with exponentially distributed single claim amounts and Erlang(2) interclaim times with $\operatorname{Erlang}(2)$ claim amounts.

Finally, considering an interest force, we consider the problem of calculating the expected discounted dividends prior to ruin, finding an integro-differential equation that they satisfy and solving it. Numerical examples are also provided for illustration.
\end{abstract}

Keywords: Sparre-Andersen risk model; $\operatorname{Erlang}(n)$ interclaim times; fundamental Lundberg's equation; generalized Lundberg's equation; probability of reaching an upper barrier; maximum severity of ruin; expected discounted dividends prior to ruin

\section{Introduction}

In the present article, we work with the Sparre-Andersen model driven by the equation

$$
U(t)=u+c t-\sum_{i=1}^{N(t)} X_{i}, \quad t \geq 0,
$$

where $u(\geq 0)$ is the initial capital, $c(\geq 0)$ is the premium income per unit time $t,\left\{X_{i}\right\}_{i=1}^{\infty}$ is a sequence of (i.i.d.) independent and identically distributed random variables, each representing a single claim amount, with common distribution function $P(x)$ and density $p(x)$. Its Laplace transform is denoted by $\hat{p}\left(\right.$.). Denote by $\mu_{k}=E\left[X_{1}^{k}\right]$ the $k$-th moment of $X_{i}$. We assume the existence of $\mu_{1}$ (this is a general condition and it is crucial for setting the positive loading factor

*Corresponding author. E-mail: alfredo@iseg.utl.pt 
assumption below). In some parts of this manuscript, we will work with cases where higher moments exist. The sequence $\left\{X_{i}\right\}$ is independent of the counting process $\{N(t), t \geq 0\}$, with $N(t)=\max \left\{k: W_{1}+W_{2}+\cdots+W_{k} \leq t\right\}$ where the random variables $W_{i}, i \in \mathbb{N}^{+}$, are i.i.d. and $\operatorname{Erlang}(n, \lambda)$ distributed, with density $k_{n}(t)$,

$$
k_{n}(t)=\frac{\lambda^{n} t^{n-1} e^{-\lambda t}}{(n-1) !}, \quad t \geq 0, \lambda>0, n \in \mathbb{N}^{+}
$$

and probability distribution function

$$
K_{n}(t)=1-\sum_{i=0}^{n-1} \frac{(\lambda t)^{i} e^{-\lambda t}}{i !}
$$

We assume a positive loading factor, that is $c E\left(W_{1}\right)>E\left(X_{1}\right) \Leftrightarrow c n>\lambda \mu_{1}$.

Moreover, the adjustment coefficient $R>0$ is the smallest positive number such that $-R$ is a solution of the Lundberg equation

$$
\left(1-\left(\frac{c}{\lambda}\right) s\right)^{n}=\hat{p}(s)
$$

Now, we set some definitions and mathematical preliminaries regarding on main objects of interest in the Sparre-Andersen model. Time to ruin is denoted as $T=\inf \{t>0: U(t)<$ $0 \mid U(0)=u\}$, and $T=\infty$ if and only if $U(t) \geq 0, \forall t>0$. The ultimate ruin probability is defined as $\Psi(u)=\operatorname{Pr}(T<\infty)$ and the corresponding non-ruin probability is $\Phi(u)=1-\Psi(u)$.

Regarding the barrier problem, which is related to the payment of dividends, we denote by $\tau_{b}=\inf \{t>0: U(t) \geq b \mid U(0)=u\}$ the first time that the surplus upcrosses the level $b \geq u$. The probability that the surplus attains the level $b$ from initial surplus $u$ without first falling below zero is given by

$$
\chi(u, b)=\operatorname{Pr}\left(T>\tau_{b} \mid U(0)=u\right),
$$

with $\xi(u, b)=1-\chi(u, b)$ being the probability that ruin occurs from $u$ before the surplus ever reaching $b$.

Assuming that the surplus process continues after ruin, we denote the time of the first upcross of the surplus through level ' 0 ' after ruin occurs by $T^{\prime}=\inf \{t: t>T, U(t) \geq 0\}$, for finite $T$. In the interval of time where the surplus is at deficit, we define the maximum severity of ruin as

$$
M_{u}=\sup \left\{|U(t)|: T \leq t \leq T^{\prime} \mid U(0)=u\right\} .
$$

The conditional distribution function of the maximum severity of ruin, given that ruin occurs, is given by

$$
J(z ; u)=\operatorname{Pr}\left(M_{u} \leq z \mid T<\infty\right), u, z \geq 0 .
$$

The probability that ruin occurs and that the deficit at ruin is at most $y$ is given by $G(u, y)=$ $P(T<\infty, U(T) \geq-y \mid U(0)=u)$. For a given $u$, this is a defective distribution function, clearly $\lim _{y \rightarrow \infty} G(u, y)=\Psi(u)$. The corresponding (defective) density is denoted as $g(u, y)$. 
The probability that the maximum deficit occurs at ruin is defined by $\operatorname{Pr}\left(M_{u}=|U(T)| \mid T<\infty\right)$. Picard (1994) showed that

$$
P\left(M_{u}=|U(T)| \mid T<\infty\right)=\frac{\int_{0}^{\infty} g(u, y) \chi(0, y) d y}{\Psi(u)} .
$$

We consider the problem where an insurance portfolio is used to provide dividend income for that insurance's company shareholders. Like before, let $u$ denote the initial surplus and let $b \geq u$ be a dividend barrier. Let the random variable $D_{u}$ denote, the present value at a positive constant force of interest per unit time of dividends payable to shareholders until ruin occurs, and denote $m$-th moment as $V_{m}(u, b)=E\left[D_{u}^{m}\right], m \geq 0$, where $V_{0}(u, b) \equiv 1$. For simplicity we will denote $V_{1}(u, b)=V(u, b)$. We assume the existence of $V_{m}(u, b)$.

In the next section, we present some of the mathematical background on the model related to our problem. In Sections 3 and 4, we study the integro-differential equation and show explicit formulas for the maximum severity of ruin. Section 5 is devoted to some particular cases where explicit expressions can easily be found. In Section 6, we give attention to the dividends problem. Finally, in the last section we state some concluding remarks.

\section{Mathematical background}

In recent years, the Sparre-Andersen model has been a major point of interest in risk theory. Many authors have done a lot of important advances in the topic. In this paper, we present some new developments.

We know from Li \& Dickson (2006) that $\chi(u, b)$ satisfies an order $n$ integro-differential equation with $n$ boundary conditions that can be written in the form

$$
B(\mathcal{D}) v(u)=\int_{0}^{u} v(u-y) p(y) d y, \quad u \geq 0,
$$

where

$$
B(\mathcal{D})=\left(I-\left(\frac{c}{\lambda}\right) \mathcal{D}\right)^{n}=\sum_{k=0}^{n}(-1)^{k}\left(\frac{c}{\lambda}\right)^{k}\left(\begin{array}{l}
n \\
k
\end{array}\right) \mathcal{D}^{k},
$$

and $\mathcal{D}$ is the differential operator. See also $\mathrm{Li}$ (2008). If we find $n$ linearly independent particular solutions $v_{j}(u), j=1, \ldots, n$ for this equation, then we have

$$
\chi(u, b)=\vec{v}(u)[V(b)]^{-1} \vec{e}^{\prime},
$$

where $\vec{v}(u)=\left(v_{1}(u), \ldots, v_{n}(u)\right)$ is a $1 \times n$ vector, $V(b)$ is a $n \times n$ matrix with entry given by

$$
(V(b))_{i j}=\left.\frac{d^{i-1} v_{j}(u)}{d u^{i-1}}\right|_{u=b}
$$

and $\vec{e}=(1,0, \ldots, 0)$ is a $1 \times n$ vector. 
In this manuscript, we will be seeking for those solutions, which in turn depend on the roots of the fundamental Lundberg's equation. Recall that the fundamental Lundberg's equation is given by

$$
\left(\frac{\lambda}{c}-s\right)^{n}=\left(\frac{\lambda}{c}\right)^{n} \hat{p}(s)
$$

We denote by the numbers $\rho_{1}, \rho_{2}, \ldots, \rho_{n-1} \in \mathbb{C}$, the roots of this equation which have positive real parts (there are of course other roots, among them is 0 and $-R$, where $R>0$ is the adjustment coefficient, see Li \& Garrido (2004)).

On the other hand, the generalized Lundberg's equation is given by

$$
\left(\frac{\lambda+\delta}{c}-s\right)^{n}=\left(\frac{\lambda}{c}\right)^{n} \hat{p}(s),
$$

where $\delta$ is a positive constant force of interest. This equation has exactly $n$ roots with positive real parts and will be considered in the section of dividends. See Li \& Garrido (2004).

$\operatorname{Li}(2008)$ finds the vector of solutions $\vec{v}(u)$ for the case when $\rho_{1}, \rho_{2}, \ldots, \rho_{n-1}$ are all distinct (in fact, all those roots are different, according to Ji \& Zhang (2011)).

Our work start, by giving an improved version for the expressions given by $\mathrm{Li}$ (2008) for the $v_{i}(u), i=1, \ldots, n$. This will be given in the next section. Then, we apply our results in order to find the corresponding expressions for the distribution of the maximum severity of ruin. Afterwards, we deal with the dividends problem, we mean the calculation of the moments $V_{m}(u, b)$. For a Poisson model, an integro-differential equation for $V(u, b)$ can be found in Dickson (2005), and for $V_{m}(u, b)$ in David et al. (2004). For the Erlang(n) model, we give the respective integro-differential equations as well as a method to find their solutions.

\section{Solutions for the integro-differential equation}

Let us consider the relation between the roots of the fundamental Lundberg's equation that have positive real parts and the solutions for the integro-differential Equation (2.1). Li (2008) found that

Theorem 3.1 If $\rho_{1}, \rho_{2}, \ldots, \rho_{n-1} \in \mathbb{C}$ are distinct, then we have the following expressions for the $v_{j}(u)$ 's

$$
\begin{aligned}
& v_{1}(u)=\Phi(u), \\
& v_{j}(u)=\sum_{i=1}^{j-1} a_{i, j} \int_{0}^{u} \Phi(u-y) e^{\rho_{i} y} d y, \quad j=2,3, \ldots, n,
\end{aligned}
$$


where $a_{i, j}=-\frac{1}{\prod_{k=1, k \neq i}^{j-1}\left(\rho_{k}-\rho_{i}\right)}, \quad i=1,2, \ldots, j-1$.

Considering our developments, we propose instead a new version of Theorem 3.1, as follows:

Theorem 3.2 If $\rho_{1}, \rho_{2}, \ldots, \rho_{n-1} \in \mathbb{C}$ are distinct, then we have the following expressions for the $v_{j}(u)$ 's

$$
\begin{aligned}
& v_{1}(u)=\Phi(u), \\
& v_{j}(u)=\int_{0}^{u} \Phi(u-y) e^{\rho_{j-1} y} d y, \quad j=2,3, \ldots, n .
\end{aligned}
$$

Proof We know from Li (2008) that any solution $v(u)$ of (2.1) has Laplace transform

$$
\hat{v}(s)=\frac{d_{v}(s)}{B(s)-\hat{p}(s)},
$$

where

$$
d_{v}(s)=\sum_{i=0}^{n-1}\left(\sum_{k=i+1}^{n}\left(\begin{array}{l}
n \\
k
\end{array}\right)\left(\frac{-c}{\lambda}\right)^{k} v^{(k-1-i)}(0)\right) s^{i} .
$$

Since $\Phi(u)$ is solution of (2.1), its Laplace transform is given by [see $\mathrm{Li}(2008)$ ]

$$
\hat{\Phi}(s)=-\Phi(0)\left(\frac{c}{\lambda}\right)^{n} \frac{\prod_{i=1}^{n-1}\left(\rho_{i}-s\right)}{B(s)-\hat{p}(s)},
$$

then we have

$$
d_{\Phi}(s)=-\Phi(0)\left(\frac{c}{\lambda}\right)^{n} \prod_{i=1}^{n-1}\left(\rho_{i}-s\right) .
$$

Now, let us see that any function $v_{j}(u)=\int_{0}^{u} \Phi(u-y) e^{\rho_{j-1} y} d y$, with $j=2,3, \ldots, n$, is solution of (2.1). We can show that

$$
B(\mathcal{D}) v_{j}(u)=d_{\Phi}\left(\rho_{j-1}\right) e^{\rho_{j-1} u}+\int_{0}^{u}(B(\mathcal{D}) \Phi(u-t)) e^{\rho_{j-1} t} d t
$$

and that

$$
\int_{0}^{u} v_{j}(u-y) p(y) d y=\int_{0}^{u}(B(\mathcal{D}) \Phi(u-t)) e^{\rho_{j-1} t} d t .
$$

Since $d_{\Phi}\left(\rho_{j-1}\right)=0, j=2, \ldots n$, we get the desired equality. It remains to prove that those $v_{j}(u)$ 's are linearly independent.

Suppose that we have a linear combination such that $\sum_{j=1}^{n} c_{j} v_{j}(u)=0, \forall u \geq 0$. Consider the cases (i) and (ii) below.

(i) $c_{1}=0$ : 
Let $H(t)=\sum_{j=2}^{n} c_{j} e^{\rho_{j-1} t}$, then

$$
\begin{aligned}
\sum_{j=1}^{n} c_{j} v_{j}(u) & =\sum_{j=2}^{n} c_{j} \int_{0}^{u} \Phi(u-y) e^{\rho_{j-1} y} d y \\
& =\int_{0}^{u} \Phi(u-y) \sum_{j=2}^{n} c_{j} e^{\rho_{j-1} y} d y \\
& =\Phi * H(u)=0 .
\end{aligned}
$$

The fact that $\Phi * H(u)=0, \forall u \geq 0$ with $\Phi(u) \not \equiv 0$, implies $H(u) \equiv 0$ almost everywhere. But $H(t)$ is a continuously differentiable function, this implies that $c_{1}=c_{2}=\cdots=c_{n}=0$.

(ii) $c_{1} \neq 0$ :

Define $G(t)=\sum_{j=2}^{n}\left(-c_{j} / c_{1}\right) e^{\rho_{j-1} t}$, so $\Phi * G(u)=\Phi(u), \forall u \geq 0$. Not all the remaining coefficients $c_{j}$ 's can be 0 , otherwise $G(t) \equiv 0$. But then $\lim _{u \rightarrow+\infty} G(u)= \pm \infty$ depending on the sign of the non zero coefficients. As $\Phi(u)$ is a non-decreasing non-negative function with $\lim _{u \rightarrow+\infty} \Phi(u)=1$, we will have that $\lim _{u \rightarrow+\infty} \Phi * G(u)= \pm \infty$, which is a contradiction.

This completes the proof.

One advantage of Theorem 3.2, is that, since for any complex root $\rho$ of the fundamental Lundberg's equation the conjugate $\bar{\rho}$ is also a root, we will have that $v(u)=\int_{0}^{u} \Phi(u-y) e^{\rho y} d y$ and its conjugate $\overline{v(u)}=\int_{0}^{u} \Phi(u-y) e^{\bar{\rho} y} d y$ are both solutions of (2.1).

Although Theorems 3.1 and 3.2 are equivalent, for large values of $n$ it is better to use Theorem 3.2 for computational purposes.

It is easy to prove that in $\operatorname{Erlang}(n)$ model we do not have possibility of multiple roots, following the argument provided by Ji \& Zhang (2011),

Theorem 3.3 In the Sparre-Andersen risk model, with interclaim times Erlang(n) distributed, the $n$ roots with positive real parts of the generalized Lundberg's Equation (2.4) are all distinct.

Proof See Ji \& Zhang (2011), page 3 .

Corollary 3.1 In the Sparre-Andersen risk model, with interclaim times Erlang(n) distributed, the $n-1$ roots with positive real parts of the fundamental Lundberg's Equation (2.3) are all distinct.

\section{The maximum severity of ruin}

In the previous section we showed how to obtain the solutions of the integro-differential equation. Now we will use these results to obtain corresponding expressions for the distribution of the maximum severity of ruin. We will find an expression for that distribution which only depends on the non-ruin probability $\Phi(u)$ and the claim amounts distribution. 
If we denote by

$$
\begin{aligned}
\vec{h}(z, u) & =\int_{0}^{z} g(u, y)\left(v_{1}(z-y), \ldots, v_{n}(z-y)\right) d y \\
& =\left(\int_{0}^{z} g(u, y) v_{1}(z-y) d y, \ldots, \int_{0}^{z} g(u, y) v_{n}(z-y) d y\right) \\
& =\left(h_{1}(z, u), \ldots, h_{n}(z, u)\right),
\end{aligned}
$$

Then from Dickson (2005) and (2.2) we know that the distribution of the maximum severity of ruin $J(z ; u)$ can be expressed as:

$$
J(z ; u)=\frac{1}{1-\Phi(u)} \int_{0}^{z} g(u, y)\left(v_{1}(z-y), \ldots, v_{n}(z-y)\right) d y[V(z)]^{-1} \vec{e}^{\prime},
$$

and we only have to find an expression for every component of $\vec{h}(z, u)$. Considering the case of the Theorem 3.2 in the previous section:

In a similar way as it is done by $\mathrm{Li}(2008)$ we get

for $j=1$ :

$$
\int_{0}^{z} g(u, y) v_{1}(z-y) d y=\Phi(u+z)-\Phi(u)
$$

and for $j=2, \ldots, n$ :

$$
\begin{aligned}
\int_{0}^{z} g(u, y) v_{j}(z-y) d y & =\int_{0}^{z} g(u, y) \int_{0}^{z-y} \Phi(z-y-x) e^{\rho_{j-1} x} d x d y \\
& =\int_{0}^{z} e^{\rho_{j-1} x}[\Phi(u+(z-x))-\Phi(u)] d x
\end{aligned}
$$

\section{Explicit expressions}

In this section, our aim is to determine explicit expressions for the (existing) moments of the maximum severity of ruin as well as the probability that the maximum severity occurs at ruin, for some cases. Li (2008) considered those moments for Erlang(2) interclaim times and exponential claims. We work here other two cases, and will be presenting formulae as well as some numerical calculations. Namely, for cases where:

(1) Interclaim arrivals are Erlang $(3, \lambda)$ and single claim amounts are Exponential $(\beta)$ distributed. For simplification, we denote this case by Erlang(3)-Exponential;

(2) Interclaim arrivals are $\operatorname{Erlang}(2, \lambda)$ and single claim amounts are $\operatorname{Erlang}(2, \beta)$ distributed. Similarly, we denote this case by $\operatorname{Erlang}(2)-\operatorname{Erlang}(2)$. 


\subsection{Erlang(3) - Exponential case}

Considering the premium per unit time $c=(1+\theta) \lambda / 3 \beta$ with safety loading coefficient $\theta>0$, the fundamental Lundberg's Equation (2.3) takes the form

$$
\left(1-\left(\frac{c}{\lambda}\right) s\right)^{3}-\frac{\beta}{(s+\beta)}=0
$$

which has four roots: $0, \rho_{1}, \rho_{2}$ and $-R$, where $0<R<\beta$ is the adjustment coefficient, $\rho_{1}, \rho_{2}$ are complex roots with positive real parts and $\rho_{2}=\overline{\rho_{1}}$. The three solutions for the integro-differential Equation (2.1) come

$$
\begin{aligned}
\Phi(u) & =1-\left(1-\frac{R}{\beta}\right) e^{-R u}, \\
v_{2}(u) & =\frac{-1}{\rho_{1}}+\frac{\beta-R}{\beta\left(R+\rho_{1}\right)} e^{-R u}+\frac{R\left(\beta+\rho_{1}\right)}{\rho_{1} \beta\left(R+\rho_{1}\right)} e^{\rho_{1} u}, \\
v_{3}(u) & =\frac{-1}{\rho_{2}}+\frac{\beta-R}{\beta\left(R+\rho_{2}\right)} e^{-R u}+\frac{R\left(\beta+\rho_{2}\right)}{\rho_{2} \beta\left(R+\rho_{2}\right)} e^{\rho_{2} u} .
\end{aligned}
$$

\subsubsection{Distribution and moments of the maximum severity}

After calculating (4.1), we get the distribution of the maximum severity of ruin

$$
1-J(z ; u)=\frac{\alpha e^{-R z}}{1-\gamma e^{-\left(\rho_{1}+R\right) z}-\delta e^{-\left(\rho_{2}+R\right) z}-\eta e^{-R z}},
$$

where

$$
\begin{gathered}
\alpha=\frac{R\left(R+\rho_{1}\right)\left(R+\rho_{2}\right)}{\beta\left(\beta+\rho_{1}\right)\left(\beta+\rho_{2}\right)} ; \gamma=-\frac{R(\beta-R)\left(R+\rho_{2}\right)}{\rho_{1}\left(\beta+\rho_{1}\right)\left(\rho_{2}-\rho_{1}\right)} \\
\delta=\frac{R(\beta-R)\left(R+\rho_{1}\right)}{\rho_{2}\left(\beta+\rho_{2}\right)\left(\rho_{2}-\rho_{1}\right)} ; \eta=\frac{(\beta-R)\left(R+\rho_{1}\right)\left(R+\rho_{2}\right)}{\beta \rho_{1} \rho_{2}}
\end{gathered}
$$

with $0<\alpha<1, \delta=\bar{\gamma}$ and $0<\eta=1-\alpha-\gamma-\delta$. Since we work with exponential claim amounts, note that this expression is independent from $u$. However, in practice, depending on the risk aversion of the insurer, the loading factor (and therefore $R$ ) might depend on the initial wealth $u$.

Consider now the moments of $M_{u}$, given that ruin occurs, the $r$-th moment is given by the formula

$$
\begin{aligned}
E\left(M_{u}^{r} \mid T<\infty\right) & =r \int_{0}^{\infty} z^{r-1}(1-J(z ; u)) d z \\
& =r \alpha \int_{0}^{\infty} \frac{z^{r-1} e^{-R z}}{1-\gamma e^{-\left(\rho_{1}+R\right) z}-\delta e^{-\left(\rho_{2}+R\right) z}-\eta e^{-R z}} d z
\end{aligned}
$$


Table 5.1. Expected values and standard deviations of $M_{u}$ for $n=1,2,3 ; m=1$.

\begin{tabular}{lllllll}
\hline$\theta$ & $n=1, m=1 E\left(M_{u}\right)$ & $s . d .\left(M_{u}\right)$ & $n=2, m=1 E\left(M_{u}\right)$ & $s . d .\left(M_{u}\right)$ & $n=3, m=1 E\left(M_{u}\right)$ & $s . d .\left(M_{u}\right)$ \\
\hline 0.05 & 3.197 & 7.324 & 2.474 & 5.532 & 2.236 & 4.933 \\
0.1 & 2.638 & 5.007 & 2.063 & 3.805 & 1.875 & 3.404 \\
0.15 & 2.342 & 4.015 & 1.848 & 3.069 & 1.687 & 2.754 \\
0.2 & 2.150 & 3.443 & 1.709 & 2.646 & 1.567 & 2.381 \\
0.25 & 2.012 & 3.064 & 1.611 & 2.368 & 1.481 & 2.136 \\
0.3 & 1.906 & 2.792 & 1.536 & 2.169 & 1.416 & 1.962 \\
\hline
\end{tabular}

for $r \geq 1$. Since $\left|\gamma e^{-\left(\rho_{1}+R\right) z}+\delta e^{-\left(\rho_{2}+R\right) z}+\eta e^{-R z}\right|<1$ we can write

$$
1-J(z ; u)=\alpha e^{-R z} \sum_{k=0}^{\infty}\left(\gamma e^{-\left(\rho_{1}+R\right) z}+\delta e^{-\left(\rho_{2}+R\right) z}+\eta e^{-R z}\right)^{k} .
$$

Hence,

$$
E\left(M_{u}^{r} \mid T<\infty\right)=\alpha r ! \sum_{k=0}^{\infty} \sum_{j=0}^{k} \sum_{l=0}^{k-j}\left(\begin{array}{c}
k \\
j
\end{array}\right)\left(\begin{array}{c}
k-j \\
l
\end{array}\right) \frac{\eta^{j} \gamma^{l} \delta^{k-j-l}}{\left(R(k+1)+\rho_{1} l+\rho_{2}(k-j-l)\right)^{r}}
$$

Choosing $\beta=1, \lambda=3$ and $c=1+\theta$, we evaluate formula (5.1) for some values of $\theta$ with $r=1$. We use the values obtained by $\operatorname{Li}$ (2008) for $n=2$ and compare them with the values which we computed for $n=3$. The purpose of this comparison is to analyse the behaviour of the moments of $M_{u}$ as $n$ increases. Figures are given in Table 5.1. From the table, we observe that the mean and the standard deviation of $M_{u}$ decrease as $\theta$ increases for the three cases. This was expected since an increase in $\theta$ means an increase in the income unit $c$, which will give faster growth of the surplus per unit of time. Also, we note that for fixed $\theta$ the mean and the standard deviation of $M_{u}$ decrease as $n$ increases. Since $E\left(W_{i}\right)=n / \lambda$, the claims arrive after longer intervals of time.

\subsubsection{The probability that the maximum severity occurs at ruin}

Due to the memoryless property of the exponential distribution we have that $g(u, y)=$ $\Psi(u) p(y)$. Hence from (1.1)

$$
P\left(M_{u}=|U(T)| \mid T<\infty\right)=\frac{\int_{0}^{\infty} g(u, y) \chi(0, y) d y}{\Psi(u)}=\int_{0}^{\infty} \chi(0, y) p(y) d y .
$$

Now from (2.2) we get, for $u=0$

$$
\chi(0, y)=\left(\frac{R}{\beta}\right) \frac{1+\frac{\rho_{1} \gamma}{R} e^{-\left(\rho_{1}+R\right) y}+\frac{\rho_{2} \gamma}{R} e^{-\left(\rho_{2}+R\right) y}}{1-\gamma e^{-\left(\rho_{1}+R\right) y}-\delta e^{-\left(\rho_{2}+R\right) y}-\eta e^{-R y}},
$$


Table 5.2. Probability that the maximum deficit occurs at ruin, for $n=3, m=1$.

\begin{tabular}{lllllll}
\hline$\theta$ & 0.05 & 0.1 & 0.15 & 0.2 & 0.25 & 0.3 \\
\hline$\tilde{p}$ & 0.735 & 0.752 & 0.768 & 0.782 & 0.795 & 0.808 \\
\hline
\end{tabular}

so

$$
\begin{aligned}
P\left(M_{u}=|U(T)| \mid T<\infty\right) & =\left(\frac{R}{\beta}\right) \int_{0}^{\infty} \frac{1+\frac{\rho_{1} \gamma}{R} e^{-\left(\rho_{1}+R\right) y}+\frac{\rho_{2} \gamma}{R} e^{-\left(\rho_{2}+R\right) y}}{1-\gamma e^{-\left(\rho_{1}+R\right) y}-\delta e^{-\left(\rho_{2}+R\right) y}-\eta e^{-R y}} \beta e^{-\beta y} d y \\
& =\int_{0}^{\infty} \frac{R+\rho_{1} \gamma e^{-\left(\rho_{1}+R\right) y}+\rho_{2} \gamma e^{-\left(\rho_{2}+R\right) y}}{1-\gamma e^{-\left(\rho_{1}+R\right) y}-\delta e^{-\left(\rho_{2}+R\right) y}-\eta e^{-R y}} e^{-\beta y} d y
\end{aligned}
$$

Choosing the same values for $\lambda, \beta$ and $\theta$ as before, we evaluate (5.2) to get the figures in Table 5.2, where $\tilde{p}=P\left(M_{u}=|U(T)| \mid T<\infty\right)$. From the table, we conclude that the probability that the maximum deficit occurs at ruin increases as $\theta$ increases. Moreover, there is small probability of getting a bigger deficit after ruin.

\subsection{Erlang(2) - Erlang(2) case}

Considering the premium $c=(1+\theta) \lambda / \beta$ with $\theta>0$, the fundamental Lundberg's Equation (2.3) takes the form

$$
\left(1-\left(\frac{c}{\lambda}\right) s\right)^{2}-\frac{\beta^{2}}{(s+\beta)^{2}}=0,
$$

which has four real roots: $0,-R_{1},-R_{2}$ and $\rho$, where $0<R_{1}<\beta$ is the adjustment coefficient and $R_{2}, \rho>\beta$.The two solutions for the integro-differential Equation (2.1) come

$$
\begin{aligned}
\Phi(u)= & 1-\frac{R_{2}\left(\beta-R_{1}\right)^{2}}{\beta^{2}\left(R_{2}-R_{1}\right)} e^{-R_{1} u}-\frac{R_{1}\left(\beta-R_{2}\right)^{2}}{\beta^{2}\left(R_{1}-R_{2}\right)} e^{-R_{2} u}, \\
v_{2}(u)= & -\frac{1}{\rho}+\frac{R_{1} R_{2}(\beta+\rho)^{2}}{\beta^{2} \rho\left(\rho+R_{1}\right)\left(\rho+R_{2}\right)} e^{\rho u}+\frac{R_{2}\left(\beta-R_{1}\right)^{2}}{\beta^{2}\left(R_{2}-R_{1}\right)\left(\rho+R_{1}\right)} e^{-R_{1} u} \\
& +\frac{R_{1}\left(\beta-R_{2}\right)^{2}}{\beta^{2}\left(R_{1}-R_{2}\right)\left(\rho+R_{2}\right)} e^{-R_{2} u} .
\end{aligned}
$$

\subsubsection{Distribution and moments of the maximum severity}

In this case, the formula obtained from (4.1) is written in the following way

$$
J(z ; u)=\frac{1}{\Psi(u)}\left[\frac{R_{2}\left(\beta-R_{1}\right)^{2}}{\beta^{2}\left(R_{2}-R_{1}\right)} e^{-R_{1} u} J_{1}(z ; u)+\frac{R_{1}\left(\beta-R_{2}\right)^{2}}{\beta^{2}\left(R_{1}-R_{2}\right)} e^{-R_{2} u} J_{2}(z ; u)\right] .
$$

So,

$$
1-J(z ; u)=\frac{1}{\Psi(u)}\left[\frac{R_{2}\left(\beta-R_{1}\right)^{2}}{\beta^{2}\left(R_{2}-R_{1}\right)} e^{-R_{1} u}\left(1-J_{1}(z ; u)\right)+\frac{R_{1}\left(\beta-R_{2}\right)^{2}}{\beta^{2}\left(R_{1}-R_{2}\right)} e^{-R_{2} u}\left(1-J_{2}(z ; u)\right)\right],
$$


where the functions $J_{1}(z ; u)$ and $J_{2}(z ; u)$ are

$$
\begin{aligned}
& J_{1}(z ; u)=\frac{1-\gamma_{1} e^{-\left(\rho+R_{1}\right) z}-\gamma_{2} e^{-\left(\rho+R_{2}\right) z}-\left(1-\gamma_{1}\right) e^{-R_{1} z}-\tau_{1} e^{-R_{2} z}-\omega_{1} e^{-\left(\rho+R_{1}+R_{2}\right) z}}{1-\gamma_{1} e^{-\left(\rho+R_{1}\right) z}-\gamma_{2} e^{-\left(\rho+R_{2}\right) z}-\delta_{1} e^{-R_{1} z}-\delta_{2} e^{-R_{2} z}-\eta e^{-\left(\rho+R_{1}+R_{2}\right) z}}, \\
& J_{2}(z ; u)=\frac{1-\gamma_{1} e^{-\left(\rho+R_{1}\right) z}-\gamma_{2} e^{-\left(\rho+R_{2}\right) z}-\tau_{2} e^{-R_{1} z}-\left(1-\gamma_{2}\right) e^{-R_{2} z}-\omega_{2} e^{-\left(\rho+R_{1}+R_{2}\right) z}}{1-\gamma_{1} e^{-\left(\rho+R_{1}\right) z}-\gamma_{2} e^{-\left(\rho+R_{2}\right) z}-\delta_{1} e^{-R_{1} z}-\delta_{2} e^{-R_{2} z}-\eta e^{-\left(\rho+R_{1}+R_{2}\right) z}},
\end{aligned}
$$

and

$$
\begin{array}{cr}
\gamma_{1}=-\frac{R_{1}\left(\beta-R_{1}\right)^{2}\left(\rho+R_{2}\right)}{\rho\left(R_{2}-R_{1}\right)(\beta+\rho)^{2}}, & \gamma_{2}=-\frac{R_{2}\left(\beta-R_{2}\right)^{2}\left(\rho+R_{1}\right)}{\rho\left(R_{1}-R_{2}\right)(\beta+\rho)^{2}}, \\
\delta_{1}=\frac{R_{2}\left(\beta-R_{1}\right)^{2}\left(\rho+R_{1}\right)}{\beta^{2} \rho\left(R_{2}-R_{1}\right)}, & \delta_{2}=\frac{R_{1}\left(\beta-R_{2}\right)^{2}\left(\rho+R_{2}\right)}{\beta^{2} \rho\left(R_{1}-R_{2}\right)}, \\
\tau_{1}=\frac{R_{1}\left(\beta-R_{2}\right)^{2}\left(\rho+R_{2}\right)}{\rho\left(R_{1}-R_{2}\right)(\beta+\rho)^{2}}, & \tau_{2}=\frac{R_{2}\left(\beta-R_{1}\right)^{2}\left(\rho+R_{1}\right)}{\rho\left(R_{2}-R_{1}\right)(\beta+\rho)^{2}}, \\
\omega_{1}=-\frac{\left(\beta-R_{2}\right)^{2}}{(\beta+\rho)^{2}}, & \omega_{2}=-\frac{\left(\beta-R_{1}\right)^{2}}{(\beta+\rho)^{2}}, \\
\eta=-\frac{\left(\beta-R_{1}\right)^{2}\left(\beta-R_{2}\right)^{2}}{\beta^{2}(\beta+\rho)^{2}}, & \alpha=\frac{R_{1} R_{2}\left(\rho+R_{1}\right)\left(\rho+R_{2}\right)}{\beta^{2}(\beta+\rho)^{2}},
\end{array}
$$

with $0<\alpha<1$ and $\eta=1-\alpha-\gamma_{1}-\gamma_{2}-\delta_{1}-\delta_{2}$.

In the same way, we compute the conditional moments of $M_{u}$, given that ruin occurs,

$$
\begin{aligned}
E\left(M_{u}^{r} \mid T<\infty\right)= & r \int_{0}^{\infty} z^{r-1}(1-J(z ; u)) d z \\
= & \frac{r}{\Psi(u)}\left[\frac{R_{2}\left(\beta-R_{1}\right)^{2}}{\beta^{2}\left(R_{2}-R_{1}\right)} e^{-R_{1} u} \int_{0}^{\infty} z^{r-1}\left(1-J_{1}(z ; u)\right) d z\right. \\
& \left.+\frac{R_{1}\left(\beta-R_{2}\right)^{2}}{\beta^{2}\left(R_{1}-R_{2}\right)} e^{-R_{2} u} \int_{0}^{\infty} z^{r-1}\left(1-J_{2}(z ; u)\right) d z\right],
\end{aligned}
$$

for $r \geq 1$.

Choosing $\beta=1, \lambda=1$ and $c=1+\theta$, we evaluate formula (5.3) for some values of $\theta$ with $r=1$. We use the values obtained by $\mathrm{Li}$ (2008) for exponential claim amounts and compare them with the values which we computed for Erlang(2) claim amounts. The purpose of this comparison is to analyse the behaviour of the moments of $M_{u}$ as $m$ increases. As before, Table 5.3 shows figures for $E\left(M_{u}\right)$ and s.d. $\left(M_{u}\right)$.

From Table 5.3 we observe that the mean and the standard deviation of $M_{u}$ decrease as $\theta$ increases for all the three cases. This was expected, since an increase in $\theta$ means an increase in the premium income $c$, which will give faster growth of the surplus, per unit of time. Note that for a fixed $\theta$ the mean and the standard deviation of $M_{u}$ are higher in the $\operatorname{Erlang}(2)-\operatorname{Erlang}(2)$ case than in the Erlang(2) - Exponential case. Since $E\left(X_{i}\right)=m / \beta$, the claims are bigger on average. 
Table 5.3. Values of $E\left(M_{u}\right)$ and s.d. $\left(M_{u}\right)$ for $n=2 ; m=1$ and $n=m=2$.

\begin{tabular}{lllll}
\hline & $n=2, m=1$ & & $n=2, m=2$ \\
$\theta$ & $E\left(M_{u}\right)$ & s.d. $\left(M_{u}\right)$ & $E\left(M_{u}\right)$ & s.d. $\left(M_{u}\right)$ \\
\hline 0.05 & 2.474 & 5.532 & 3.279 & 7.137 \\
0.1 & 2.063 & 3.805 & 2.759 & 4.911 \\
0.15 & 1.848 & 3.069 & 2.485 & 3.959 \\
0.2 & 1.709 & 2.646 & 2.307 & 3.411 \\
0.25 & 1.611 & 2.368 & 2.179 & 3.049 \\
0.3 & 1.536 & 2.169 & 2.082 & 2.791 \\
\hline
\end{tabular}

Table 5.4. Probability that the maximum deficit occurs at ruin, for $n=2, m=2$.

\begin{tabular}{lllllll}
\hline$\theta$ & 0.05 & 0.1 & 0.15 & 0.2 & 0.25 & 0.3 \\
\hline$\tilde{p}$ & 0.730 & 0.745 & 0.759 & 0.772 & 0.784 & 0.795 \\
\hline
\end{tabular}

\subsubsection{The probability that the maximum severity occurs at ruin}

From (2.2) we get, for $u=0$

$\chi(0, y)=\left(\frac{R_{1} R_{2}}{\beta^{2}}\right) \frac{1+\frac{\rho \gamma_{1}}{R_{1}} e^{-\left(\rho+R_{1}\right) y}+\frac{\rho \gamma_{2}}{R_{2}} e^{-\left(\rho+R_{2}\right) y}}{1-\gamma_{1} e^{-\left(\rho+R_{1}\right) z}-\gamma_{2} e^{-\left(\rho+R_{2}\right) z}-\delta_{1} e^{-R_{1} z}-\delta_{2} e^{-R_{2} z}-\eta e^{-\left(\rho+R_{1}+R_{2}\right) z}}$.

The formula for $P\left(M_{u}=|U(T)| \mid T<\infty\right)$ is obtained in the same way as in Equation (5.2). Choosing the same values of $\lambda, \beta$ and $\theta$ as before we evaluate that probability to get the figures in Table 5.4 where $\tilde{p}=P\left(M_{u}=|U(T)| \mid T<\infty\right)$. From the table, we conclude that the probability that the maximum deficit occurs at ruin increases along with $\theta$. Moreover, there is small probability of getting a bigger deficit after ruin. Comparing Tables 5.2 and 5.4 we can see that for higher values of $n$, the probability of falling to lower levels of deficit after ruin is smaller.

\section{Dividends}

In this section, we consider the dividends problem. We can use the method by Dickson \& Waters (2004) to generalize an equation for $V_{m}(u, b)$ in $\operatorname{Erlang}(n)$ risk process. So, conditioning on the time and the amount of the first claim we get, for $0 \leq u<b$

$$
\begin{aligned}
V_{m}(u, b)= & \int_{\frac{b-u}{c}}^{\infty} k_{n}(t) e^{-m \delta t}\left[\left(c \bar{s} \overline{t-\frac{b-u}{c}}\right)^{m}\right. \\
& \left.+\sum_{j=1}^{m}\left(\begin{array}{c}
m \\
j
\end{array}\right)\left(c \bar{s} \frac{b-u}{t-\frac{b-u}{c}}\right)^{m-j} \int_{0}^{b} f(x) V_{j}(b-x, b) d x\right] d t \\
& +\int_{0}^{\frac{b-u}{c}} e^{-m \delta t} k_{n}(t) \int_{0}^{u+c t} V_{m}(u+c t-x, b) p(x) d x d t, \quad m \geq 1 .
\end{aligned}
$$


In particular, for $m=1$

$$
\begin{aligned}
V(u, b)= & \int_{\frac{b-u}{c}}^{\infty} k_{n}(t) e^{-\delta t}\left(c \bar{s} \frac{\overline{b-u}}{c}+\int_{0}^{b} f(x) V(b-x, b) d x\right) d t \\
& +\int_{0}^{\frac{b-u}{c}} e^{-\delta t} k_{n}(t) \int_{0}^{u+c t} V(u+c t-x, b) p(x) d x d t,
\end{aligned}
$$

where $\bar{s}_{t\rceil}=\frac{e^{\delta t}-1}{\delta}$ in standard actuarial notation.

For an $\operatorname{Erlang}(n)$ risk process, the integro-differential equations satisfied by the discounted expected dividends are

$$
\begin{aligned}
\left(\left(1+\frac{\delta}{\lambda}\right) \mathcal{I}-\frac{c}{\lambda} \mathcal{D}\right)^{n} V(u, b) & =\int_{0}^{u} V(u-x, b) p(x) d x \\
\left.\frac{d^{k} V(u, b)}{d u^{k}}\right|_{u=b} & =\left(\frac{\delta}{c}\right)^{k-1}, 1 \leq k \leq n,
\end{aligned}
$$

and for a general $m$

$$
\begin{aligned}
\left(\left(1+\frac{\delta}{\lambda}\right) \mathcal{I}-\frac{c}{\lambda} \mathcal{D}\right)^{n} V_{m}(u, b) & =\int_{0}^{u} V_{m}(u-x, b) p(x) d x \\
\left.\frac{d^{k} V_{m}(u, b)}{d u^{k}}\right|_{u=b} & =\sum_{j=1}^{k} \frac{m !}{(m-j) !}\left\{\begin{array}{l}
k \\
j
\end{array}\right\}\left(\begin{array}{l}
\delta \\
c
\end{array}\right)^{k-j} V_{m-j}(b, b), 1 \leq k \leq n,
\end{aligned}
$$

where $\left\{\begin{array}{l}k \\ j\end{array}\right\}=\frac{1}{j !} \sum_{i=0}^{j}(-1)^{j-i}\left(\begin{array}{l}j \\ i\end{array}\right) i^{k}$ denotes the Stirling numbers of the second kind. We define for convenience $V_{m-j}(u, b) \equiv 0$, for $m<j$ in the formula above.

These equations generalize those proposed by Dickson (2005) and Dickson \& Waters (2004) for the classical Poisson risk model, and are the same equations as proposed by Albrecher et al. (2005).

Following an argument originally proposed by Bühlmann (1970), Section 6.4.9 for a Poisson risk model, we propose for an $\operatorname{Erlang}(n)$ risk model that $V(u, b)$ can be written in the form

$$
V(u, b)=\sum_{i=1}^{n} C_{i} e^{\rho_{i} u} \beta_{i}(u),
$$

where $C_{i}$ 's are constants (that depend on the parameter $b$ ), $\rho_{i}$ 's are the $n$ roots with positive real parts of the generalized Lundberg's Equation (2.4), and the functions $\beta_{i}(u)$ are solutions of

$$
\left(\lambda_{i} \mathcal{I}-c \mathcal{D}\right)^{n} \beta_{i}(u)=\lambda_{i}^{n} \int_{0}^{u} \beta_{i}(u-x) p_{i}(x) d x
$$

with $\lambda_{i}=\lambda \hat{p}^{\frac{1}{n}}\left(\rho_{i}\right)$ and $p_{i}(x)=\frac{e^{-\rho_{i} x} p(x)}{\hat{p}\left(\rho_{i}\right)}$. 
The constants $C_{i}$ 's are determined using the boundary conditions given in (6.3), which gives a system of $n$ equations with $n$ unknowns

$$
\left.\frac{d^{k} V(u, b)}{d u^{k}}\right|_{u=b}=\left.\sum_{i=1}^{n} C_{i} \frac{d^{k}\left(e^{\rho_{i} u} \beta_{i}(u)\right)}{d u^{k}}\right|_{u=b}=\left(\frac{\delta}{c}\right)^{k-1}, 1 \leq k \leq n,
$$

It can be written in matrix form as

$$
\left(\begin{array}{c}
C_{1} \\
C_{2} \\
\vdots \\
C_{n}
\end{array}\right)=\left(\left.\begin{array}{cccc}
\left.\frac{d\left(e^{\rho_{1} u} \beta_{1}(u)\right)}{d u}\right|_{u=b} & \left.\frac{d\left(e^{\rho_{2} u} \beta_{2}(u)\right)}{d u}\right|_{u=b} & \cdots & \left.\frac{d\left(e^{\rho_{n} u} \beta_{n}(u)\right)}{d u}\right|_{u=b} \\
\left.\frac{d^{2}\left(e^{\rho_{1} u} \beta_{1}(u)\right)}{d u^{2}}\right|_{u=b} & \left.\frac{d^{2}\left(e^{\rho_{2} u} \beta_{2}(u)\right)}{d u^{2}}\right|_{u=b} & \cdots & \frac{d^{2}\left(e^{\rho_{n} u} \beta_{n}(u)\right)}{d u^{2}} \\
\vdots & \vdots & \ddots & \vdots \\
\frac{d^{n}\left(e^{\rho_{1} u} \beta_{1}(u)\right)}{d u^{n}} & \left.\frac{d^{n}\left(e^{\rho_{2} u} \beta_{2}(u)\right)}{d u^{n}}\right|_{u=b} & \cdots & \left.\frac{d^{n}\left(e^{\rho_{n} u} \beta_{n}(u)\right)}{d u^{n}}\right|_{u=b}
\end{array}\right|^{-1}\left(\begin{array}{c}
1 \\
\left(\begin{array}{c}
\delta \\
c
\end{array}\right) \\
\vdots \\
\left(\frac{\delta}{c}\right)^{n-1}
\end{array}\right)\right.
$$

We summarize this in the following theorem:

Theorem 6.1 The solutions of integro-differential Equation (6.3) are of the form

$$
V(u, b)=\sum_{i=1}^{n} C_{i} e^{\rho_{i} u} \beta_{i}(u),
$$

where $\rho_{i}$ 's are the roots with positive real parts of the generalized Lundberg's Equation (2.4), $\beta_{i}(u)$ 's are defined in (6.6) and the constants $C_{i}$ 's are defined in (6.7).

Proof The proof is straightforward and follows by taking derivatives of $V(u, b)$ and finding out which conditions must be satisfied by the $\rho_{i}$ 's and $\beta_{i}(u)$ 's to get the equality in (6.3).

This method generalizes the results of Albrecher et al. (2005), since it works for any kind of claim amounts distribution, and not only for the distributions with rational Laplace transforms.

Special care should be taken in the case some of the roots ( $\rho_{i}$ 's) of the generalized Lundberg's equation are complex, by using standard techniques of the theory of differential equations.

The same approach can be implemented to find a general $V_{m}(u, b), m \geq 2$, writing it in the form (6.5) and using the corresponding boundary conditions given in (6.4).

\subsection{Example}

In the following example, we compute $V(u, b)$ and $V_{2}(u, b)$ for the Erlang(2) risk model with Erlang(2) claim amounts.

Let the interclaim times $W_{i}$ and the claim amounts $X_{i}$ be both $\operatorname{Erlang}(2,2)$, let the positive loading $c=1.1$ and the force of interest $\delta=0.03$.

- $V(u, b)$ : 
Table 6.1. Values of $V(u, b)$ for $0 \leq u, b \leq 9$.

\begin{tabular}{llllllllll}
\hline$b \backslash u$ & 0 & 1 & 2 & 3 & 4 & 5 & 6 & 7 & 8 \\
\hline 0 & 1.064 & & & & & & & & \\
1 & 0.836 & 1.808 & & & & & & & \\
2 & 0.856 & 1.847 & 2.846 & & & & & \\
3 & 0.848 & 1.828 & 2.815 & 3.803 & & & & \\
4 & 0.801 & 1.728 & 2.661 & 3.597 & 4.574 & & & \\
5 & 0.730 & 1.575 & 2.424 & 3.277 & 4.174 & 5.143 & & \\
6 & 0.648 & 1.397 & 2.151 & 2.908 & 3.705 & 4.575 & 5.538 & \\
7 & 0.565 & 1.218 & 1.875 & 2.535 & 3.229 & 3.988 & 4.840 & 5.010 & 5.967 \\
8 & 0.486 & 1.049 & 1.615 & 2.184 & 2.782 & 3.436 & 4.170 & 5.285 & 5.118 \\
9 & 0.416 & 0.897 & 1.381 & 1.867 & 2.379 & 2.938 & 3.566 & 6.073 \\
\hline
\end{tabular}

We get $\rho_{1}=0.169, \rho_{2}=2.631$,

$$
\begin{aligned}
& \beta_{1}(u)=1+0.026 e^{-2.954 u}-0.718 e^{-0.492 u}, \\
& \beta_{2}(u)=1+0.047 e^{-5.235 u}-0.108 e^{-3.845 u},
\end{aligned}
$$

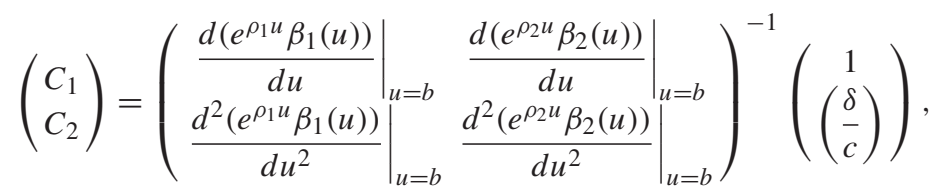

so

$$
\begin{aligned}
& C_{1}=C_{1}(b)=\frac{0.323 e^{7.123 b}-0.163 e^{8.512 b}+6.849 e^{12.358 b}}{d(b)} \\
& C_{2}=C_{2}(b)=\frac{-0.205 e^{6.942 b}+0.081 e^{9.404 b}-0.024 e^{9.896 b}}{d(b)}
\end{aligned}
$$

where

$$
\begin{aligned}
d(b)= & 0.002 e^{4.337 b}-0.015 e^{5.727 b}+0.065 e^{6.799 b}+0.057 e^{7.291 b} \\
& -0.027 e^{8.189 b}-0.031 e^{8.681 b}-1.039 e^{9.572 b}+1.802 e^{12.034 b}+1.093 e^{12.526 b},
\end{aligned}
$$

and

$$
V(u, b)=C_{1} e^{\rho_{1} u} \beta_{1}(u)+C_{2} e^{\rho_{2} u} \beta_{2}(u) .
$$

The values on the Table 6.1 are identical to the ones obtained by Albrecher et al. (2005).

- $V_{2}(u, b)$ :

We get $\rho_{1}=0.273, \rho_{2}=2.654$,

$$
\begin{aligned}
& \beta_{1}(u)=1+0.033 e^{-3.054 u}-0.636 e^{-0.673 u}, \\
& \beta_{2}(u)=1+0.047 e^{-5.256 u}-0.107 e^{-3.873 u}
\end{aligned}
$$


Table 6.2. Values of $V_{2}(u, b)$ for $0 \leq u, b \leq 9$.

\begin{tabular}{lllllllllll}
\hline$b \backslash u$ & 0 & 1 & 2 & 3 & 4 & 5 & 6 & 7 & 8 & 9 \\
\hline 0 & 1.709 & & & & & & & & & \\
1 & 2.239 & 5.230 & & & & & & & \\
2 & 3.512 & 7.865 & 12.910 & & & & & & \\
3 & 4.193 & 9.376 & 15.184 & 21.977 & & & & & \\
4 & 4.192 & 9.375 & 15.176 & 21.870 & 30.042 & & & & \\
5 & 3.763 & 8.416 & 13.622 & 19.630 & 26.947 & 36.132 & & & \\
6 & 3.163 & 7.075 & 11.452 & 16.503 & 22.656 & 30.410 & 40.300 & & & \\
7 & 2.556 & 5.717 & 9.254 & 13.335 & 18.308 & 24.578 & 32.632 & 42.990 & & \\
8 & 2.017 & 4.511 & 7.302 & 10.522 & 14.445 & 19.393 & 25.754 & 34.008 & 44.669 & \\
9 & 1.569 & 3.509 & 5.680 & 8.184 & 11.236 & 15.085 & 20.033 & 26.460 & 34.844 & 45.697 \\
\hline
\end{tabular}

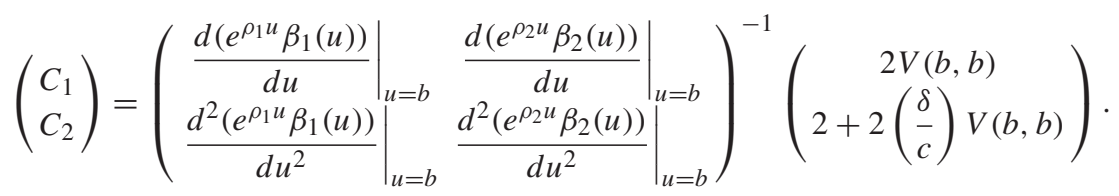

The expressions for $C_{1}=C_{1}(b)$ and $C_{2}=C_{2}(b)$ are obtained in the same way as those for $V(u, b)$.

Finally we get

$$
V_{2}(u, b)=C_{1} e^{\rho_{1} u} \beta_{1}(u)+C_{2} e^{\rho_{2} u} \beta_{2}(u),
$$

and the table of values for $V_{2}(u, b)$ is as follows

The values on the table are identical to those obtained by Albrecher et al. (2005).

\section{Some concluding remarks}

In this work we have shown, based on the techniques provided by $\mathrm{Li}$ (2008), a new method to find expressions for the distribution of the maximum severity of ruin in the Sparre-Andersen model with Erlang $(n)$ interclaim times. Those expressions depend exclusively on the non-ruin probability and the claim amounts distribution.

In a Sparre-Andersen model with $\operatorname{Erlang}(n)$ distributed interclaim times, the expected times between claims are larger for higher values of $n$, therefore the moments of the maximum severity of ruin are smaller.

The probability that the maximum severity occurs at the moment of ruin is bigger for higher values of $n$. If we want to obtain similar explicit formulas for higher values of $n$, the computations will become quite messy. However, we can still obtain numerical results using software like Mathematica.

In the case of Erlang $(m)$ distributed claim amounts, the expected sizes of the claims are larger for higher values of $m$, and therefore the moments of the maximum severity of ruin are also higher. 
We generalized the results obtained by Albrecher et al. (2005) to find the expected present value of dividends for any arbitrary claim amount distribution.

\section{Acknowledgements}

The authors gratefully acknowledge financial support from FCT-Fundação para a Ciência e a Tecnologia (Grant reference BD 67140/2009 and programme FEDER/POCI 2010).

\section{References}

Albrecher H., Claramunt M. M., \& Mármol M. (2005). On the distribution of dividend payments in a Sparre-Andersen model with generalized Erlang $(n)$ interclaim times. Insurance: Mathematics \& Economics 37, 324-334.

Bühlmann, H. (1970). Mathematical methods in risk theory. Berlin: Springer-Verlag.

Dickson, D. C. M. (2005). Insurance risk and ruin. Cambridge University Press.

Dickson, D. C. M. \& Waters, H. R. (2004). Some optimal dividends problems. Astin Bulletin 34, 49-74.

Ji, L. \& Zhang, C. (2011). Analysis of the multiple roots of the Lundberg fundamental equation in the PH(n) risk model. Applied Stochastic Models in Business and Industry.

$\mathrm{Li}, \mathrm{S}$. (2008). A note on the maximum severity of ruin in an $\operatorname{Erlang}(n)$ risk process. Bulletin of the Swiss Association of Actuaries, 167-180.

Li, S. \& Dickson, D. C. M. (2006). The maximum surplus before ruin in an $\operatorname{Erlang}(n)$ risk process and related problems. Insurance: Mathematics \& Economics 38 (3), 529-539.

Li, S. \& Garrido, J. (2004). On ruin for the Erlang(n) risk process. Insurance: Mathematics \& Economics 34 (3), 391-408.

Picard, P. (1994). On some measures of the severity of ruin in the classical Poisson model. Insurance: Mathematics \& Economics 14, 107-115. 